REPORT OF GENERAL DISCUSSIONS AT IAU SYMPOSIUM NO. 78 ON NUTATION AND THE EARTH'S ROTATION

G. A. Wilkins (with the assistance of R. J. Dejaiffe and

R. Verbeiren)

\title{
Introduction
}

The purpose of this report is to summarize the discussions held on Friday, 1977 May 27, that preceded the adoption by IAU Symposium No. 78 of the four symposium resolutions concerning the theoretical and observational studies of nutation. It was the aim in the first half of the session to clarify the scientific problems and to obtain a broad concensus so that the resolutions committee could finish drafting the resolutions to be put to the symposium after the coffeebreak. During the second half-session some new scientific points were made, but they have been incorporated in the report of the discussions during the first half-session; the report for the second half-session contains only a record of the voting and of the discussions on procedure and on the wording of the resolutions.

The Chairman of the session was G. A. Wilkins and the co-chairman was V. K. Abalakin, who from time to time summarized in Russian the discussions that had taken place in English, and vice-versa. This report is based mainly on manuscript notes made by the Chairman, and on extensive notes which were sent to him by R. J. Dejaiffe and R. Verbeiren; amendments suggested by members of the Organizing Committee have been included. Comments and questions have not always been attributed to individual participants, but rather an attempt has been made to summarize the outcome of the discussions that led to the final form of the resolutions.

The initial discussions concerned two main topics: the choice of the axis to which the ephemeris of the forced nutation should refer and the requirements for an improved series for computing the ephemeris. The controversy on these topics was such that there was insufficient time to discuss thoroughly the desirability of introducing new terminology for coordinate systems for describing the rotation of the Earth. 
Choice of axis for nutation

The Chairman pointed out that when the IAU (1976) system of astronomical constants was adopted at Grenoble it was agreed that the ephemeris of the "... nutation shall include the forced periodic terms listed by Woolard for the axis of figure in place of those given for the instantaneous axis of rotation ...," but Fedorov had proposed that the theory of the precession and nutation should describe the motion of the axis of angular momentum of the whole Earth. In the free nutation of the rigid Earth the axes of angular momentum (A), rotation (R) and figure $(F)$ are coplanar, and the ratio FA:AR is about $300: 1$, but the actual positions of $F$ and $R$ are not predictable since they depend on unknown sources of excitation and damping of the motion. The separation of A and $\mathrm{R}$ is less than 0".001. If there were no free nutation the axes of rotation and figure would be displaced from the axis of angular momentum by predictable amounts to the varying positions $R^{\prime}$ and $F^{\prime}$, respectively, where $R^{\prime}$ is the position now tabulated and $F^{\prime}$ is the position to be tabulated in accordance with the IAU (1976) recommendation. Again the ratio $F^{\prime} A: A^{\prime}$ for a rigid Earth would be about $300: 1$. The angular separation of $A$ and $F^{\prime}$ would be only about 0".006 while that of $A$ and $F$ varies around $0 ! ! 2$; thus, the forced luni-solar component of the polar motion is very much smaller than the free nutation.

The Chairman then asked E. P. Fedorov to comment on the choice of the axis to which the theory of the forced nutation should refer. He first of all remarked that there is a fourth axis, namely that through the conventional international origin (CIO), which is defined by the adopted positions of the international latitude stations; and which would be fixed with respect to $F$ if the Earth were rigid. He suggested that what is needed is the motion of the axis through the CIO relative to a non-rotating frame of reference, but it appears to be necessary to use one of the possible intermediate coordinate systems. He considered that the best choice would be the total angular momentum vector of the Earth, for the following reasons. Firstly, he claimed that the motion of this vector is practically independent of all the properties of the Earth other than its moments of inertia. Secondly, he considered that, at least in the case of the rigid Earth, the observations of the zenith distances of the meridian crossings of circumpolar stars are related to the angular momentum vector rather than to the instantaneous axis of rotation. He said that he was encouraged in this opinion by the remarks made by Sir Harold Jeffreys in the foreword to his (i.e., Fedorov's) book on nutation.

The first of these reasons was challenged by Melchior who pointed out that the viscoelastic properties of the Earth are such that there is a phase lag between the tidal bulge and the perturbing body, and so the torque does depend on the non-rigidity of the Earth. Fedorov considered that the effect is small. (There was further discussion on this point in the later discussion on the adoption of new coefficients.) Melchior also commented, however, that the pole of a star catalogue is very close to the axis of angular momentum, and so his 
personal preference would be for this axis rather than for the axis of figure. Guinot considered that there was little to choose between $A$ and $R$, but that we should not use the axis of figure since the significance of the point $F^{\prime}$ is not easily defined. Bender suggested, however, that $F^{\prime}$ may be interpreted as being the position of the axis of the figure if there were no free nutation, and that the advantage of its use is that geophysicists will not have to subtract this forced contribution to the motion of $\mathrm{F}$.

The Chairman suggested as a compromise that the main tabulations of nutation should give the motion of the axis of the angular momentum, $A$, as suggested by Fedorov, but that in addition the displacement $A^{\prime}$ should also be tabulated. Melchior explained how the series for the displacements of $R^{\prime}$ and $F^{\prime}$ from $A$ are related to the series for the tidal forces, taking into account the effect of aliasing; the terms of the series for $\mathrm{AF}^{\prime}$ are sometimes known as diurnal nutations or the Oppolzer terms. Guinot supported the suggestion that the diurnal nutations should be listed separately, and further support came when others pointed out that these terms are considerably affected by the departures of the Earth from the rigid model adopted in Woolard's theory. The discussion about the magnitude of the effects of non-rigidity was inconclusive, although Bender suggested that the actual displacement of $R^{\prime}$ from A, as discussed by McClure, is considerably greater than that given by rigid-body theory.

The second of Fedorov's reasons for preferring the axis of angular momentum was also discussed. For example, it was suggested that since the observations are made from the Earth's surface it would be appropriate to use the angular momentum vector for the mantle only and not that for the whole Earth, but the discussion did not lead to any clear conclusions. Later, Mikhailov spoke strongly in favor of the view that we should continue to adopt the conventional concept of the true pole that it is the instantaneous axis of rotation of the Earth. Melchior and Mulholland also considered that this axis will continue to be the simplest in use.

\section{The series for the nutation}

The Chairman opened the discussion on the adoption of a new series for nutation. He pointed out that it is necessary to decide whether the urgency of the need for an improved series is such that empirical corrections should be applied to the present series or whether it would be better to wait until a new theory which takes into account the viscoelastic properties of the Earth could be developed.

Yatskiv first of all said if the nutation were to refer to the axis of total angular momentum it would only be necessary to change the coefficient of the principal term, but he then went on to suggest the adoption of a new set of coefficients which would refer to the mean axis of figure of the mantle. He discussed the coefficients which would be 
amended and claimed that the following new values would be in accord with both observations and the predictions of Molodensky's second model.

\begin{tabular}{ccc}
\hline Period & in $\Delta \varepsilon$ & in $\Delta \psi \sin \varepsilon$ \\
\hline 18.6 years & $9 . ! 206$ & $-6 . " 843$ \\
1.0 years & 0.005 & +0.057 \\
0.5 years & 0.572 & -0.523 \\
13.7 days & 0.097 & -0.090 \\
\hline
\end{tabular}

Mulholland questioned the first statement, but Yatskiv confirmed it and said that the coefficient of the principal term should be changed from 9".210 to 9".229. Melchior said that in order to make Woolard's theory consistent with values of the other constants that were adopted in 1976 it would be necessary to change the coefficient to 9".2293. He considered, however, that the adopted coefficients should represent the actual motion of the astronomical instruments which are connected to the crust on the mantle. It is not possible to specify the nutation by one coefficient since the amplitudes of the terms are modified by resonance effects which depend upon frequency.

Fedorov repeated his view that the motion of the total angular momentum vector is independent of the model and that the effects of the liquid core are reflected in the correction terms required to represent the motion of the axis through the CIO. Mulholland considered that it would not be useful to adopt two lists of coefficients, but it would be better to adopt a single list of coefficients to represent the observed nutation.

Melchior suggested that it would be better to use the word "experimental" rather than "empirical" since the proposed coefficients are based on observational data and are consistent with the theory of Molodensky. He considered that it would be appropriate to submit such a list of coefficients to the relevant IAU commissions for adoption. In response to questions he claimed that the errors of the coefficients would be not more than 1 or 2 in units of $0: 001$. Bender thought that this did not include the effect on the rotation axis pointed out by McClure. Mulholland remarked that the currently adopted values are certainly wrong, but that it will be necessary to wait for 18.6 years before it will be possible to know whether the proposed values are wrong. He held that there is an urgent need for a revised nutation series which will be in better accord with both observations and theoretical calculations which allow for the elasticity of the mantle and the fluidity of the core. Krasinsky, on the other hand, considered that it will be better to continue to use a series based on the theory of rotation of a rigid Earth. 